\title{
Erratum to: Geochemical behavior and environmental risks related to the use of abandoned base-metal tailings as construction material in the upper-Moulouya district, Morocco
}

\author{
R. Argane ${ }^{1,2} \cdot$ M. El Adnani ${ }^{4}$ - M. Benzaazoua ${ }^{1,3} \cdot$ H. Bouzahzah ${ }^{3} \cdot$ A. Khalil $^{4}$. \\ R. Hakkou ${ }^{2}$ - Y. Taha ${ }^{3}$
}

Published online: 31 October 2015

(C) Springer-Verlag Berlin Heidelberg 2015

Erratum to: Environ Sci Pollut Res

DOI 10.1007/s11356-015-5292-y

The original version of this article contains a mistake.

The correct Chatain et al. 2013 reference should be:

Chatain V, Benzaazoua M, Loustau Cazalet M, Bouzahzah H, Delolme C, Gautier M, De Brauer C (2013) Mineralogical study and leaching behavior of a stabilized harbor sediment with hydraulic binder. Environ Sci Pollut Res 20(1):51-59.

The online version of the original article can be found at http:dx.doi.org/ 10.1007/s11356-015-5292-y.

R. Argane

rabei.argane@gmail.com

1 Laboratoire de Génie Civil et d'Ingénierie Environnementale, Université de Lyon, INSA — Lyon, 20 av. A. Einstein, 69621 Villeurbanne Cedex, France

2 Laboratoire de Chimie desMatériaux et de l'Environnement, Faculté des Sciences et Techniques, Université Cadi Ayyad, 40000 Marrakech, Morocco

3 UQAT, 445 Boulevards de l'université, Rouyn-Noranda, Québec, Canada

4 Ecole Nationale Supérieure des Mines de Rabat Avenue, Hadj Ahmed Cherkaoui BP 753, Agdal, Rabat, Morocco 